\title{
Research Progress on Detection Methods of Food Additives in Sports Health Food
}

\author{
Peishen Zhang ${ }^{*}$
}

Xianren Rd $1^{\text {st }}$ Section, Youxian, Mianyang, Sichuan, Mianyang Normal University, China

\begin{abstract}
With economic development and continuous improvement in the of living standards, people's demand for food has increased. In order to improve the quality of food such as its taste, appearance, and texture, food processing industries have started to develop synthetic substances which are added to food, known as food additive. There is a wide variety of food additives making different effects on the human body, and some even seriously endanger consumers' health, especially sportspersons, as they need healthier more after exercise. Therefore, the detection and management of food additives are necessary, which can be managed by introducing the principles of classification and usage of food additives, carrying out routine inspection and testing technologies, introducing systems of analysis of food additive, technology used for detection and methods of application, and by taking corresponding improvement measures.
\end{abstract}

Keywords: Food safety, hybrid intelligent system food additive, sports.

\section{INTRODUCTION}

Food additives are chemical synthetics or natural substances added to food in order to improve the quality, color, aroma, taste and requirements of antisepsis and processing of food. . Food additives are basically synthetic [1-3] therefore, excessive use can cause varying degrees of harm to humans. The Standardization Committee of food additives was established in 1980 in China which made provisions regarding the application and use of additives. However, because of residues of additives in the food, technological development lags behind the developments in the food additives industry. Illegal production of food additives and their excessive use are still very serious issues. To strengthen the study on the detection of food additives, qualitative and quantitative assessment will be extremely important $[4,5]$.

\section{MATERIAL AND METHODS}

\subsection{Snapshot of the Development of Food Additives in China}

Comprehensive, systematic research and management of food additives in China although started late, but it developed rapidly [1].

First stage: Soon after the establishment of the new China, provisions were made to abandon the use of certain additives in the production of food,.

Second stage: In 1973, the National Health Standard for Food Additives established a research collaboration group, and began a comprehensive study on the issues related to food additives. In 1977, it was promulgated by the National Health Standard for the use of food additives and the food additive regulation on health at the start of the comprehensive management.

*Address correspondence to these authors at the Xianren Rd $1^{\text {st }}$ Section, Youxian, Mianyang, Sichuan, Mianyang Normal University, China; Tel: +358-6-3247476; Fax: +358-6-3247457; E-mail: hunter2011@foxmail.com
Third stage: In 1980, an organization was set up named as a "National Food Additive Standards Technical Committee", for the standardization and internationalization of China's food additives. Since then, the development of China's food additives industry has been rapid $[6,7]$.

Today, food additives have emerged as a new discipline, and have become one of the basic industries categorized as the modern food industry.

\subsection{Advantages and Disadvantages of Food Additives}

Food industry never experiences decline. At present, China ranks first in generating output in all sectors of the food industry, with the growth rate much higher than the national economic growth. Food additive in food production is the most active and most creative element, and is the soul of the food industry. Food plays an important role in promoting industrial development. Lack of food additives implies lack of modern food industry. Although they are added in food only to $0.01 \% 10$, ten-zeros, however, this is enough to improve the color, aroma and taste, for adjusting the structure of food nutrition, improving food quality and food processing conditions. Food additives also play an important role in the extension of food shelf life, as well as for comprehensive utilization of food resources. If there are no modern food additives, there would be lack of modernization of the food industry [8].

Beneficial effects of food additives: Food additives have contributed greatly to the development of the food industry, and are known as the soul or pillars of the modern food industry. Among several benefits they bring to the food industry, their main function is as follows. Firstly, they are conducive to the preservation of food, prevention of food spoilage and enhancement of the quality of food. Secondly, they improve the organoleptic properties of foods to meet people's requirements of food's flavor, color and texture. Thirdly, they maintain or improve the nutritional value of food, which 
can enable the food industry to save resources and at the same time, significantly improve the quality of food to increase their added value, in order to achieve significant economic and social benefits.

Furthermore, they have, increased the variety of food meeting the varied needs of consumers. [9-11] They have also enabled the food processing and manufacturing processes as being more rational, healthier, and convenient, for industrial mechanization, automation and standardization of food industry.

The safety problem of food additives: Food additives play an important role in the development of food industry, but the use of food additives also has its principles, namely, safety and efficacy, with safety being more important. GB2760 11,996 national mandatory standards of hygiene provide standards for using food additives with specific titles, classification, use, and dosage of food additives. [12, 13]. At present, in China, mainly the following issues exist regarding the use of food additives:

1) Excessive use of food additives. There has been an extension in the limit of use of food additives, which has not only resulted in substandard products, but also has threatened human health.

2) Super scope of use of food additives. Excessive use of food additives is beyond the scope of mandatory national standards required by certain food additives that can be used in specific type and within the scope. Alcohol, fragrance, saccharin and water" blended wines fall into severe cases which have exceeded the scope of use .

3) Use of unauthorized or prohibited additives. These substances are not approved by the national food hygiene departments or have been explicitly labelled as banned additives, which once again have been added to food products. This will inevitably lead to the poisoning or even death of food stuffs safety accidents. 2008 San Lu milk powder adds a "melamine".

4) Use of substandard, fake, and expired food additives. Food additive products have certain quality requirements. Unqualified food additives are mainly embodied in product purity. Some bad food additives contain small amount of mercury, lead, arsenic and other toxic and harmful substances, which seriously affect the quality of products, thus, endangering consumers' health.

\subsection{The Development Trend of Food Additives}

Now countries are committed to developing new kind of food additives and food additive fabrication technologies. The development trend of food additives is as follows: First, the research and development of natural food additives. Second is the rigorous study on bio-food additive. Thirdl is the creation of a new food additive synthesis. Fourth, involves studying food additive compound and other applications; Fifth includes research-specific functional food additives. Sixth is the study on polymeric additives and seventh is the active development of preservatives and preservation technology.

\subsection{Food Safety and Inspection}

Food is the material basis for human survival and development, and food security is the major issue related to human health and livelihood. "Food security" refers to: "on food by its original uses for production or edible Shi does not on consumers caused damage of a guarantees. Security of food stresses on the idea that food should not damage or threat human's health. It is a concept of science, which can also be expressed as security involved in " planting of food, farming, processing, packaging, transport, storage, and sales activities, meeting mandatory standards . Absolute security is impossible, therefore, generally relative safety of food is taken into consideration so that it does not lead to health damage under normal consumption practices. Effective control measures of food entail eliminating hazardous substances or toxic substances to ensure food security, which primarily depend on food preparation with respect to food quantity a number of other internal factors.

In recent years, frequent serious incidents have been reported in some regions and countries in the world. China's food safety issues have also been significant. With rapid economic and social development in China, obtaining basic solution of the problem of food security causing health problem, has become a serious concern, especially in China. as WTO of members, and world States of trade from will increasingly increase, food security has become no borders, world a area of food problem is may will spread global, thus on China food security brings huge effects. Food safety problems to a certain extent, affect agricultural products in China and the strategic adjustment of industrial structure.

The idea of food security implies that food does not contain substances or factors that may harm or threat human health. If food safety is guaranteed, it implies monitoring of food safety, quality, damage or health-threatening substances, and other factors for inspection. Meanwhile, for the assessment of safety, detection methods are used to provide a scientific basis for the assessment. Therefore, assessment not only plays an important role in food safety management, but is also indispensable for proposing in food safety precautions.

In order to ensure food safety, it requires a supervision management, using fast and effective means of analysisIn addition, it should assure satisfactory consumption environment for consumers, guarantee security of people's health., control not security of food entered or as less of entered people of diet among, effective guarantees China of food security, is Government and research workers, and producer, and operators, and managers zanier of society are in concern of focus matters.

\section{CATEGORIES OF FOOD ADDITIVES}

Food additives are divided into 23 categories according to their functionality, and standards of usage which include 2,400 varieties of food additives. In addition, China currently allows the use of food nutrition fortification agents involving about 200 species.

Food additives are classified according to their origin, method of production, the role and function, which are different for different divisions. 
According to the origin, there are two categories of food additives; natural food additives and artificial chemical compounds. Natural food additives are extracted from plants and animals produced by biotechnological methods by either fermentation or enzymatic hydrolysis. Food additives obtained by chemical synthesis can be divided into general and synthesizing natural equivalents, such as natural spices, natural pigments, etc. Furthermore, naturally extracted additives are classified into three categories according to the production methods, chemical synthesis, and biosynthesis (enzymatic and fermentation).

Classification by role and function: In 1990, China provided classification codes for classifying food additives according to their main function. Food additives were classified into; acidity regulation agent, anti-knot agent, elimination bubble agent, anti-oxidant, bleach, expanded pine agent, glue Sam sugar based agent, colouring agent, nursing colour agent, emulsion Agent, enzyme preparations, increased flavour agent, flour processing agent, film agent, and water keeping agent, nutrition strengthened agent, preservatives, stable and solidified agent, sweet agent, increased heavy agent, food with spice, and food industrial with processing additives and the other additives.

\section{DETECTION TECHNOLOGY AND METHODS}

\subsection{Artificial Pigment}

High performance liquid chromatography has become the main method for the detection of artificial pigment, which is also the first method GB/T 5009 35-2003(for the determination of synthetic colorants in food), due to their separation ability. DAD combined with variable wave length detectors allowed the detection of more targeted food additives. Liu fen, introduced high performance liquid chromatography combined with DAD detector on lemon yellow, amaranth, cochineal and sunset yellow and bright blue method. Chromatographic column EC ODS is $51 \mathrm{um}(125 \mathrm{~min} * 4 \mathrm{~mm}) \mathrm{mo}-$ bile phase methanol. $0.02 \mathrm{~mol} / 1$ ammonium acetate and methanol gradient: after the initial $10 \%, 3 \mathrm{~min}$ to $30 \%, 5 \mathrm{~min}$ to $70 \%$ for $1 \mathrm{~min}$. In variety of different synthetic pigments peak time. The best detection wave length was selected which can greatly improve the detection sensitivityand overcome at $254 \mathrm{~nm}$ the baseline wanders caused by gradient Elution. Accuracy improved the quantitative. There are other methods of paper chromatography, Xi oscilloscope spectroscopy, UV spectrophotometry, and thin-layer chromatography.

There is a sample method known as high performance liquid chromatography sample preparation method, which includes simple matrix samples, which if frozen, become carbonated and alcoholic.

Beverage Direct water samples can be dissolved, after being transferred to suitable $\mathrm{pH}$ value followed by the determination of the membrane with constant volume. For a more complex sample matrix, juice can be used.

In the polyamide adsorption method, the heated sample solution and sodium citrate $\mathrm{pH}=6$ are mixed with polyamide powder. Water-soluble impurities are removed by Filtering Wash, and methanol and formic acid wash away natural pigment: washed. Be neutral. With ammonia --desorption pigment ethanol, steamed after the removal of ammonia solution of constant volume. The Audi acidic pigment of the Mono-ago structure falls yellow, lemon yellow, Carmine, allure red etc. and methylene biphenyl brilliant blue Indigo. Indigo tribe has strong adsorption capacity. Then use sulphuric acid- sodium tungstate precipitate proteins. Or you can ether was used to remove the oil, and then use the ultrasonic extraction.

\subsection{Food Sweet Agent}

Common sweeteners include; sodium saccharin, sugar, and asparagine Acicula fame potassium. -Phenylalanine methyl ester (Sweeteners), and others are mostly used in high performance liquid chromatography and also reported to be used in ion chromatography. G Jian Hua, enhanced 'GB/T5009. 97-2003 discarded by capillary column gas chromatography for determining sodium cyclamate in yellow rice wine by chromatographic column CP Sile $5 \mathrm{CB}$ $(30 \mathrm{~m} * 0.25 \mathrm{~mm} * 0.25 \mathrm{um})$. Detector FID, tested room temperature of 200 degrees Celsius. Inlet temperature, was 200 degrees Celsius. Carrier gas (high pure nitrogen) flow rate 1 $\mathrm{mL}$ in 1 minute. Hydrogen flow rate $30 \mathrm{~mL} / \mathrm{min}$, air velocity $300 \mathrm{~mL} / \mathrm{min}$. Tail blown $29 \mathrm{~mL} / \mathrm{min}$, injection volume $1.0 \mathrm{~L}$, split ratio $1 / 10$ (initially), $1 / 20 \quad(0 \mathrm{~min}), 1 / 100 \quad(0.75$ min). Column box temperature 100 degrees Celsius for 4 min. With speed of 50 degrees Celsius /min up to 150 degrees centigrade. Hold for 5 minutes.

Sample pre-treatment methods: Gas chromatography method was used for the determination of sodium cyclamate, taking advantage of the decomposition of sodium nitrite of sodium cyclamate in food. Cyclohexane under acidic conditions was produced by esterification into volatile nitrites of Cyclohexane by gas chromatographic method. The samples were placed in an ice-water bath, and sodium nitrite and sulphuric acid solution were added. The samples were mixed well, and placed in ice water bath for some time, followed by the addition of n-hexane and sodium chloride. By oscillating static hierarchy, n-hexane extract was obtained.

In high performance liquid chromatography, since most of the sweeteners are soluble in water, therefore, for some matrix simple foods, like carbonated beverages, and alcoholic beverages (prior to the removal of carbon dioxide and ethyl alcohol), sweeteners were only extracted by diluting them in a certain volume of water. For a more complex sample matrix, like milk beverages, and soy sauce, water and ultrasonic extraction were added, which were mixed with protein precipitants, and were added into the filter through filtrate membrane.

For solid samples, for ultrasonic extraction, inater was added into the membrane constant volume.

\subsection{Food Antioxidants}

Currently, high performance liquid chromatography is widely used. It can simultaneously determine multiple antioxidants. Hu X Z by reversed-phase high performance liquid chromatographic method determined 9 kinds of phenolic antioxidants in oil using methanol-acetic acid-water system for a mobile phase, using gradient elution. Determination 
was carried out in a linear range of $1 \sim 200 \mathrm{mg} / \mathrm{L}(\mathrm{R}=0$. 99850.9997 ), with the detection limit of $2 \mathrm{mg} / \mathrm{kg}$, and the recovery rate was $82.4 \%$ to $98.7 \%$. RSD was $1.1 \%$ to $14.74 \%$. Lin Anting used liquid chromatography-tandem mass spectrometry method for the simultaneous detection of matrix components, and more complex BHA indeed, OG, PG, TBHQ, NDGA, and DG6 seed antioxidants. Detection limits for $2 \mathrm{mg} / \mathrm{kg}$ were qualitatively in line with EU rules. Residue analysis of linear, recoveries and precision met the requirements. In addition, there is another methods named as gas chromatography, for colorimetric determination of antioxidants.

Sample pre-treatment methods employing high performance liquid chromatography were prompted. Generally, non-polar solvents such as hexane, and ether were extracted and the polarity solvent such as acetonitrile was distributed in liquid . For the preparation of polar solvent, rotary steam volume concentration membrane was measured using gas chromatography method. Generally, it is dissolved with hexane oil, extracted with acetonitrile and ethanol blends. The solvent is removed after TMS treatment for testing.

\subsection{Food Preservatives}

In recent years, for the determination of preservatives in foods, gas chromatography and high performance liquid chromatography are used for simultaneous determination of many kinds of preservatives including 8 food preservatives for DE oxidation acetic acid, propionic acid, sorbet acid, benzoic acid, hydroxyl benzoic acid methyl ester and ethyl hydroxyl benzoic acid, p-hydroxyl benzoic acid, $\mathrm{N}$ and hydroxyl benzoic acid butyl ester. Detection limit was 1.5, 1.0, $1.5,1.0,2.0,2.0,2.0$, and $2.0 \mathrm{mg} / \mathrm{L}$ 。 respectively. Wu Y J established solid phase extraction and high performance liquid chromatographic method for the determination of dehydrogenation acid, benzoic acid and sorbet acid in pastry method. In the $0.02-0.15 \mathrm{mg} / \mathrm{mL}$, adequate linear relationships were observed. RSD was $1.0 \%$ to $2.5 \%$. Recovery was 96. $2 \%-102.1 \%$, as the method is simple, rapid and sensitive, with good precision and accuracy. In addition, capillary electrophoresis detection of preservatives in foods was also reported.

Sample pre-treatment methods: Preparation of gas chromatography sample is as follows: the matrix of food (such as vinegar, juice,) was added in hydrochloric acid $(1+1)$, by acidification, extracted with ethyl ether and washed with acid solution of sodium chloride. Following this, it formed a anhydrous sodium sulphate layer. Petroleum ether was ether-dissolved. For the complex matrix of foods, (such as meat, and hot sauce), the current measurement method is used for the determination of interference in food ingredients to be removed first. For example, potassium cyanide-zinc acetate as a precipitant, sodium hydroxide - zinc sulphuric acid as precipitant, benzoic acid and sorbet acid soluble in water in alkaline environment. Characteristic of acidic environments is that do not dissolve in water, under alkaline conditions to remove grease, then measured with simple matrix method for similar foods.
High performance liquid chromatographic method was used for sample preparation: For the matrix of food (soft drinks, fruit juices, wine), specimens can be dissolved in water, by adjusting $\mathrm{pH}$ value at a constant volume after centrifugation. On the complex matrix of foods: (such as pastries, sauces, etc.), interference in food ingredients is also removed first. Pan Qing reported that benzoic acid and sorbet acid in acidic conditions can be distilled with steam. The method is simple, fast, and involves a variety of complex matrices of benzoic acid and sorbet acid in foods.

\subsection{Simultaneous Determination of Various Food Addi- tives}

Food can sometimes contain different types of food additives. Development in the methods for simultaneous determination of various food additives will greatly increase the efficiency of the examination, but for a more complex food matrix, because of different nature of additives reports were presented based on simultaneous determination of various food additives by high performance liquid chromatography which were relatively similar to the simultaneous determination of preservatives and sweeteners. Extraction of the sample involved two steps, extraction and mixing for simultaneous determination of various foods.

Huang established a high performance liquid chromatographic method, for simultaneous determination of 8 preservatives in soy sauce or drink and 3 kinds of sweeteners containing domain's new testing method. ODS-C18 column, with $20 \mathrm{mmol} / \mathrm{L} \mathrm{KH} 2 \mathrm{P} 04$ and acetonitrile as the mobile phase gradient, with a UV detection wavelength: $230,254 \mathrm{~nm}$ and 2 . $50-162 \mathrm{ug} / \mathrm{mL}$ linear range. $\mathrm{R}=0.999$. Recovery rate was $97.2 \% \sim 199.2 \%$, and the method was highly sensitive but reliable.

Sample pre-treatment methods are used in high performance liquid chromatography, as most preservatives and sweeteners are soluble in water. Simultaneous determination of 2 kinds of food additives, involves simple food matrix (wine, soda, etc.) which can be simply extracted or diluted in a certain amount of water. For more complex food matrix (soy sauce, milk, cakes, etc.) water or ultrasonic extraction is required, for mining protein precipitation agent and solid phase extraction column purification technology.

\section{EXPERIMENT AND CONCLUSION}

Experiment condition: The conditions included, mobile phase methanol: 20mmol.L-1, acetate buffer solution (pH6.8), gradient Elution (methanol:10\% 4min;10\%$20 \%, 20 \% / \mathrm{min}, 20 \%$ keep $2 \mathrm{~min} ; 20 \%-30 \%, 6.7 \% / \mathrm{min}$, and $30 \%$ kept for $3 \mathrm{~min}$; $30 \%-95 \%, 21.7 \% / \mathrm{min} ; 95 \%-$ $10 \%, 85 \% / \mathrm{min}$, and $10 \% \mathrm{kept}$ for $2 \mathrm{~min}$, (details are shown in Table 1) and the flow velocity was $1.0 \mathrm{ml} / \mathrm{min}$, with column temperature of 30 celsius. Sample size was $10 \mathrm{uL}$, with ultraviolet detectors for detection in $234 \mathrm{~nm}$ mand quantifying peak area. By using peak area of concentration in drawing, the regression equation was solved. 


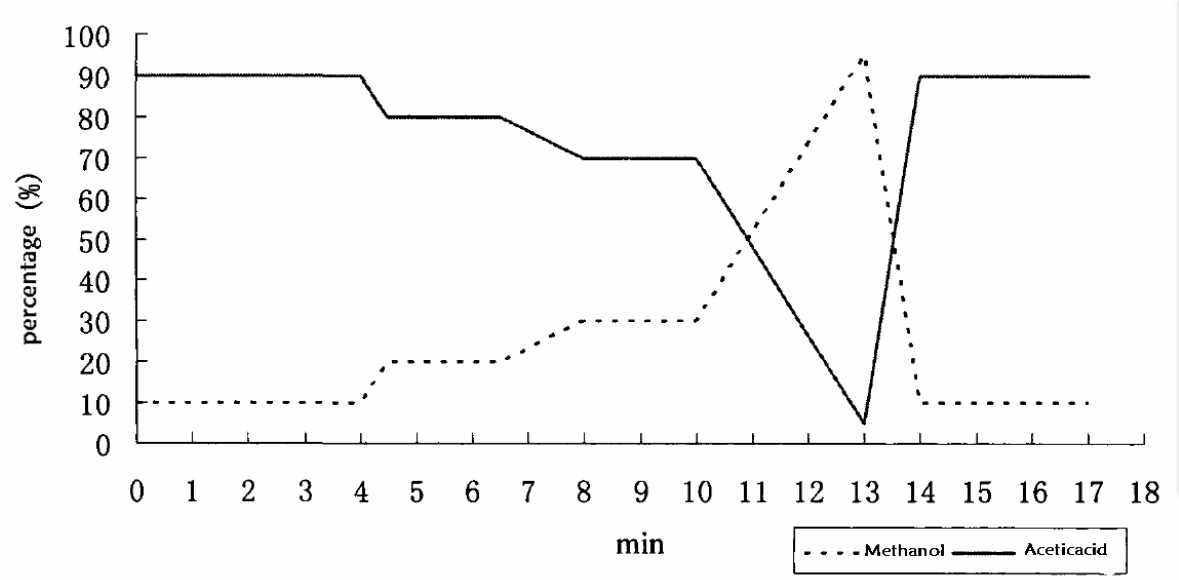

Fig. (1). Acetic acid and methanol over time curves.

Table 1. Gradient elution ratio.

\begin{tabular}{|c|c|c|}
\hline Time & $\begin{array}{c}\text { (Acetic acid) A-phase } \\
\text { Proportion (\%) }\end{array}$ & $\begin{array}{c}\text { (Methanol) B-phase } \\
\text { Proportion (\%) }\end{array}$ \\
\hline \hline 4 & 90 & 10 \\
\hline 4.5 & 90 & 10 \\
\hline 6.5 & 80 & 20 \\
\hline 8 & 80 & 20 \\
\hline 11 & 70 & 30 \\
\hline 14 & 70 & 30 \\
\hline 15 & 5 & 95 \\
\hline 17 & 90 & 10 \\
\hline
\end{tabular}

Experimental study was based on mobile phase composition and proportion, buffer $\mathrm{pH}$, temperature, flow rates and other factors affecting the separation. These make the optimum conditions define optimal elution conditions. Using methanol and acetic acid as mobile phase system reduced the cost and toxicity to extract different kinds of preservatives, sweeteners, and for simultaneous determination of synthetic colours. Two sets of gradient elution procedure were created in this paper. Gradient Elution shortened the time of analysis and increased the degree of separation, improved peak shape and detection sensitivity. After screening, wavelength was determined as $234 \mathrm{~nm}$ under $254 \mathrm{~nm}$, with each component giving a better response, effectively improving the sensitivity and overcoming the baseline shift, while reducing the interference of coexistence.

Acetic acid and Methanol over time curves are as show in Fig. (1).

To sum up, detection technology and methods of food additives have developed very fast in recent years. Chromatographic techniques have become an important means of detection of food additives. But most of the methods generally have the following problems: 1 . Food matrices are complex. Almost all kinds of analysis and testing methods of sample pre-treatment are slightly cumbersome, and are directed at the specific substrate, employing a method of applying narrow Fan Z X, Audi.2. There are a variety of test methods used for the determination of additives in food . Although some methods can perform simultaneous detection of several additives, but in a limited range. 3 chromatographic methods are the most widely used methods, but they cannot be relied upon to confirm the results. Simplified sample preparation procedures, focusing on the development of various food additives as well as the determination methods and $\mathrm{GC} / \mathrm{MS}$, LC/MS/MS final results confirmed by study on detection of food additives should be given great attention.

\section{CONFLICT OF INTEREST}

The author confirms that this article content has no conflict of interest.

\section{ACKNOWLEDGEMENTS}

This work was supported by the Key Project of Guangxi Social Sciences, China (No. gxsk201424), the Education Science fund of the Education Department of Guangxi, China (No.2014JGA268), and Guangxi Office for Education Sciences Planning, China (No.2013C108).

\section{REFERENCES}

[1] R. Brooks, and M. Kaupp, "ORCA: a component model and repository”, Software engineering for experiment robotics, pp 231-251, 2007.

[2] D. M. Gilmour, M. R. Davey, and E. C. Cocking, "Plant regeneration from cotyledon protoplasts of wild Med / c species," Plant Science, vol. 48, pp. 107-112, 1987.

[3] Z. Ge, and H. Lin, "Rubber Cambodia determined by thin-layer chromatography of synthetic colorants in food and capsule," Journal of Instrumental Analysis, vol. 12, no. 3, pp. 45-48, 1993.

[4] M. Lu, "Modern testing technology and its application in food safety," Anhui Agricultural Sciences, vol. 35, no. 21, pp. 65896590, 2007. 
[5] C. Y. Li, "Crystal of benzoic acid and sorbet acid in meat by Gas chromatographic method for the determination," Chinese Journal of Public Health, vol. 22, no. 5, pp. 609, 2006.

[6] X. Liu, Y. Ba, P-black acid, "Sorbic acid and sacchar in food separation effect of different chromatographic determination," Chinese Journal of Health laboratory, vol. 16, no. 2, pp. 242, 2006.

[7] L. Liu, J. Li, and G. Fen, "Ultraviolet Spectrophotometric simultaneous determination of Tart and sunset yellow," Spectroscopy laboratory, vol. 24, no. 3, pp. 423-427, 2007.

[8] D. Y. Lu, M. R. Davey, and E. C. Cocking, "A comparison of the cultural behave of protoplasts From leaves, cotyledons and roots of Medico sativa," Plant Science letters, vol. 31, pp. 87-99, 1983.

[9] Y. Tang, and W. P. Zhang, "High performance liquid chromatographic method for the determination of jelly Colors," Health research, vol. 31, no. 1, pp. 69-70, 2001.
[10] G. Yang, "Study on determination of synthetic colors in soft drinks," Food Industry Branch Science And Technology, vol. 23, no. 3, pp. 66-67, 2002.

[11] P. Zhang, and H. Tan, "High performance liquid chromatographic determination of saccharin sodium in food and glucose as," Chinese Journal of Health laboratory, vol. 10, no. 5, pp. 522-523, 2000.

[12] Z. Y. Zhang, "High performance liquid chromatographic method for the determination of meat, poultry, and dairy products towel pigment Study," Chinese Journal of health laboratory, vol. 16, no. 8, pp. 928, 2006.

[13] J. Zou, W. Chen, and J. Shao, "High performance liquid chromatographic method for the determination of population of synthetic pigments in fruit juice imports Inspection and quarantine, vol. 1, no. 1 , pp.26-27, 2001.

Received: June 10, 2015

Revised: July 29, 2015

Accepted: August 15, 2015

(c) Peishen Zhang; Licensee Bentham Open.

This is an open access article licensed under the terms of the (https://creativecommons.org/licenses/by/4.0/legalcode), which permits unrestricted, noncommercial use, distribution and reproduction in any medium, provided the work is properly cited. 